\title{
Tecnología en el aula infantil. Apuntes y comentarios
}

\section{Using technology in the early childhood classroom: Some comments and observations}

\author{
Wilma ROBLES-MELÉNDEZ \\ Abraham S. Fischler School of Education
}

Recibido: Diciembre 2011

Aceptado: Marzo 2012

\section{Resumen}

En este artículo se discute la presencia y rol de las tecnologías en el aula infantil dentro del contexto y expectativas de la sociedad actual. Se examina su necesidad y justificación dentro de las experiencias del aula del nivel infantil tomando en cuenta el concepto de las prácticas apropiadas. Se establece su justificación a través del examen de varios estudios que describen los beneficios derivados como resultados del uso de las tecnologías con niños pequeños. También se discute el rol del educador en el buen uso y aplicación dentro de las experiencias del aula infantil. Se destaca la necesidad de ofrecer al educador infantil formación en el uso de las tecnologías como uno de los aspectos para propiciar su integración en el aula. Se ofrecen una serie de sugerencias para su uso y práctica en el aula infantil.

Palabras clave: educación infantil; tecnología; prácticas apropiadas, niños y tecnología; computadoras y niños.

\begin{abstract}
This article discusses the role of technology in the early childhood classroom considering current societal needs and expectations. Rationale for its use is presented from the perspective of developmentally appropriate practices for children ages birth-8. Its role in the early childhood classroom is supported through the discussion of findings from several research studies. The role of the early childhood educator is discussed as a factor for appropriate classroom use. The need to prepare early childhood teachers in the use of technology is discussed as a factor to support its integration in the classroom. Suggestions for use of technology as part of early childhood practices are presented.
\end{abstract}

Keywords: early childhood education; technology; developmentally appropriate practices; children and technology, children and computers. 
Son las diez de la mañana y en el salón de cuatro años, dos niños comparten animadamente un libro interactivo que al tacto repite frases del cuento. Mientras, en el rincón de computadoras, junto a su maestra tres niños interesados en conocer más sobre las animales de la selva exploran y comentan sobre las imágenes que encontraron en una página de Internet. Más tarde en el área de bloques uno de los niños tomará fotos con la cámara digital de la construcción realizada por sus compañeros. Orgullosos verán como la maestra desplegará las fotos de su trabajo en el aula así como incluirá copias de las mismas en sus portafolios de progreso.

En muchos lugares imágenes como la anterior son ya hoy comunes donde ya no sorprende ver a los niños interactuar y utilizar las tecnologías. Muchos comienzan en el hogar donde se inician como testigos de su uso por los adultos así como partícipes viendo las tecnologías como un elemento más de su realidad. Solo basta recordar los programas de la televisión educativa como Plaza Sésamo que en casa disfrutan los pequeños y el cual desde su comienzo hace más de tres décadas ha propiciado el aprendizaje inicial. Los vemos hoy utilizar los teléfonos inteligentes con gran destreza mientras otros dibujan usando una tableta electrónica. Del hogar al aula la presencia de las tecnologías es visiblemente observable en muchos lugares aunque no siempre así en el caso del aula infantil. A pesar de ser ya esta una experiencia característica de las vivencias directas e indirectas de los niños, todavía encontramos que su práctica e integración en la sala del nivel infantil es motivo de gran discusión y vacilación. Muchos consideran que el dilema sobre su uso tal vez surge por el hecho de considerar a las tecnologías aún como algo novedoso revelando dudas y confusión sobre su función y beneficios particularmente en el nivel infantil (Lisenbee, 2009; Turja, Endepohls-Ulpe y Chatoney, 2009). Algunos aducen su exclusión al hecho de considerarlas como algo ajeno a la forma de aprender de los niños lo cual es refutable si bien reconocemos que en todo momento son un recurso más con las cuales cuenta el educador. Considerando el compromiso educativo de capacitar al niño para el éxito, en este trabajo reflexionamos sobre las razones que apoyan el uso de las tecnologías como elemento pedagógico así como los factores para su integración como parte de las experiencias formales de los niños de cero a ocho años.

\section{Educar con miras al éxito}

Si educar es preparar para el éxito dentro del marco de una sociedad, visto desde este ángulo, entendemos porque ya, al considerar el compromiso y responsabilidad del proceso educativo, no podemos excluir la necesidad de capacitar al educando para ser un buen conocedor y usuario de las tecnologías. Definimos tecnologías como todos aquellos recursos digitales y electrónicos que incluyen equipos, computadoras, software, recursos, herramientas así como el acceso a la Internet que sirven para apoyar y fomentar el proceso de enseñar y aprender (International Society for Technology in Education [ISTE] 2011; National Association for the Education of Young Children [NAEYC], 2011, 2012). Son estas un variado recurso en manos del maestro que sabiamente planifica su utilización con la intención de apoyar las experiencias de aprendizaje Contrario a lo que muchos puedan pensar su fin es contribuir al proceso de en- 
señanza y no el sustituir al maestro sobre del cual depende su buen uso y propósitos en el aula.

Hoy, el inventario de materiales y equipos didácticos va más allá de las computadoras incluyendo una creciente variedad en la serie de artefactos digitales y tecnológicos ya disponible para uso en el aula así como personal. De igual forma, según se multiplican las tecnologías, su lugar en el aula se reafirma al abrir estas nuevas oportunidades para su aplicación en el ámbito educativo tanto para el estudiante como para los educadores. Durante la reciente década se han multiplicado las aplicaciones, herramientas digitales y recursos disponibles a través de Internet dirigidos a los niños preescolares y de primaria que sirven para apoyar el aprendizaje. Prueba de esto lo son las páginas de Internet con aplicaciones interactivas que contribuyen entre otras al desarrollo de las destrezas de lectoescritura y también las pizarras inteligentes (smart board). Al igual encontramos la variedad de aplicaciones de asistencia para estudiantes con necesidades especiales cuyo uso positivo ya ha sido validado a través de varios estudios (Lau, Higgins, Hong y Miller, 2005).

De igual forma las tecnologías son hoy un valioso recurso para el educador que busca ampliar y refinar sus conocimientos. Los llamados webinars, blogs, wikis y cursos virtuales forman parte del repertorio de actividades profesionales que permiten para mantener al maestro al día sobre los estudios y prácticas pedagógicas. Mucho más, ya hoy es común encontrar programas de formación pedagógica con componentes en línea así como otros en muchos casos que ofrecen la titulación totalmente de forma virtual.

\section{Tecnologías y educación: En el aula infantil}

¿Computadoras, tecnología en el aula infantil? Como dijéramos antes para algunos su necesidad es todavía cuestionable viéndolas a veces como algo ajeno dentro del contexto de la educación temprana. Muchos continúan debatiendo su función y aún más cuando hablamos del nivel infantil. Ciertamente entendemos la preocupación expresada por muchos sobre su uso e impacto en el proceso de desarrollo particularmente cuando su empleo no está de acuerdo a las necesidades de los pequeños. Uno de estos es el caso del número de horas que invierten los niños frente al televisor o computadora. Datos de la Asociación Americana de Pediatría (2011) alertan que en promedio los niños y adolescentes están más de siete horas frente a las pantallas. En la mayoría de los casos el tiempo invertido fuera de servir como diversión carece de propósito específico o de supervisión por un adulto. Mientras existen y se reconocen las valiosas aportaciones de la televisión educativa así como de los muchos recursos y programas y software educativos, para que el mismo rinda beneficios su finalidad, regulación y supervisión es imprescindible tanto en el hogar como en el aula (NAEYC, 2012; O’Keefe y Clark-Pearson, 2011; Strassburger, 2010).

El buen uso y propósito definido subraya toda práctica en el nivel infantil, detalle que describe el empleo de las tecnologías en la sala de los niños pequeños. Cuando conceptualizamos el aula como escenario donde los pequeños exploran, aprenden y adquieren las experiencias y destrezas que les servirán para ser parte de la sociedad actual, no hay duda de la necesidad de contar con las tecnologías como parte del sinnúmero de actividades de la sala infantil. Visto como otro elemento dentro de la varie- 
dad de actividades que viene a ampliar, apoyar y crear interés respetando las formas de aprender de los niños, es así como ya hoy vemos que la oportunidad para su empleo efectivo abarca la esfera infantil. Considerando las experiencias y nociones que sobre las tecnologías poseen los niños, las mismas sirven en el aula como recurso para apoyar su interés mientras se explora de forma activa y construyen nuevos conceptos e ideas (NAEYC, 2012; Lisenbee, 2009). Con el docente como mediador y coparticipante en actividades comunes como la lectura con libros digitales, juegos en las computadoras, búsquedas de datos así como en la selección de aquellos a utilizar, su uso y lugar en el aula infantil se integra como parte de las experiencias formativas de los niños en la sociedad actual (NAEYC, 2011).

\section{En la práctica: Uso de las tecnologías en el aula infantil}

Como testigos y partícipes directos e indirectos para los niños hoy el uso de tecnologías se suma al tropel de expectativas y parámetros que su sociedad espera estos adquieran. La realidad social del contexto actual, en la segunda década del siglo, claramente indica vivimos en un mundo en gran medida condicionado por y dependiente de las tecnologías. Por eso su presencia en el aula se justifica como elemento clave que permite preparar al niño como usuario en un mundo en continua evolución tecnológica. Los resultados sobre su empleo en la práctica del aula infantil sirven como justificante para su integración. Así lo revelan las investigaciones realizadas que aunque escasas puntualizan sus importantes beneficios (Clements y Sarama, 2003).

Según revelan varios informes la exposición e interacción con las tecnologías ya sea la televisión, las computadoras o los juegos digitales se cuentan dentro de las experiencias comunes que caracterizan a la niñez en la actualidad (Rideout, Vandewater, y Wartella, 2003). Basta observar a los niños para ver como estos con más frecuencia integran la presencia de la tecnología en su hablar, formas de comunicarse, y particularmente en el juego dramático. No sorprende ver a los pequeños utilizar un objeto para representar un teléfono celular, una tableta (similar al IPad) imitando así lo que ven hacer a los adultos o en los medios. De hecho, en observaciones realizadas en una muestra de programas para niños de tres y cuatro años, se encontró que, en la mayoría de los rincones de juego dramático que incluían algún tipo de artefacto tecnológico, ya fueran modelos de juguete u objetos reales en desuso donados al aula, los mismos eran frecuentemente utilizados por los niños como parte de sus dramatizaciones (Robles de Meléndez y Valdés, 2008). De especial interés resultó ver como en su representación y dramatización se fomentaba un intercambio lingüístico que sin duda contribuye positivamente al desarrollo de un concepto emergente sobre lo que es comunicación. Esto coincide con los resultados encontrados en varios estudios revelando que cuando su uso es adecuado según las necesidades y características de los pequeños y teniendo en cuenta la participación e intervención del maestro las tecnologías tienen beneficios positivos en el desarrollo cognitivo así como social (NAEYC, 2011; Lee, 2009; Lau, Higgins, Hong, y Miller, 2005; Judge, 2005; Haugland, 1999). 


\section{Lo que nos dicen las investigaciones}

Un vistazo a varios estudios realizados sobre el uso de las tecnologías indica que las mismas pueden contribuir de manera positiva en el aprendizaje de los niños. Sin duda entre lo que conocemos sobre su impacto hay que incluir el uso de tecnologías más comunes como lo es la televisión educativa cuya programación ha servido para estimular el aprendizaje en el área de lectoescritura, y matemáticas por décadas (Thakkar, Garrison y Christakis, 2006; Fisch, 2004). Programas como Plaza Sésamo o Between the Lions [Entre los leones] han demostrado su influencia en el la adquisición de habiliades del área de lectoescritura igualmente como en las destrezas prosociales (Zerh, 2009; Fisch, 2004; Fisch y Truglio, 2001; Strickland y Rath, 2000).

Los hallazgos de varios estudios donde se investigó la efectividad de una variedad de tipos de tecnología también dejan ver sus efectos positivos en el ámbito escolar. En un estudio realizado con niños de cinco años donde medió el uso de software educativo para el apoyo de las destrezas de lectoescritura y matemáticas se encontró que el mismo propició ganancias positivas en ambas áreas (Judge, 2005). La efectividad de las tecnologías como medio en el aprendizaje de un segundo idiomas demostró resultados positivos con un grupo de niños de primaria donde el uso de multimedia propiciara la comprensión así como la adquisición de una mejor pronunciación (Esthet-Alkalat y Chajut, 2007). Respecto al uso de las tecnologías como factor en el aprendizaje se ha encontrado que el uso de software, equipos digitales y computadoras en la sala de niños de cuatro a seis años contribuye a crear y reforzar el interés de los niños sobre los temas a explorar. Vistas las tecnologías como un recurso que les permite conocer más, sin duda su empleo contribuye al proceso de construcción de ideas (Cause y Chen, 2010; Lisenbee, 2009; Hinchliff, 2008; Judge, 2005). Otros estudios han determinado su efectividad en el aprendizaje de las destrezas de apresto en particular en el área de lenguaje con niños de tres y cuatro años (Feng y Benson, 2007). Subrayamos que los resultados son evidentes cuando se emplean de forma apropiada lo cual discutimos aquí, detalle que en común también concluyen las investigaciones realizadas.

\section{Los niños como usuarios}

Según apuntáramos anteriormente al llegar al aula ya muchos pequeños traen consigo un variado repertorio de experiencias con la tecnología las cuales van desde el conocimiento incipiente a su uso como práctica regular en el ámbito familiar (Johnson, 2011; Judge, 2005). Su continuada exposición y aprendizaje en el uso de las nuevas tecnologías viene a ser responsabilidad y a tener lugar en el ámbito del aula infantil. Como un continuo de experiencias formativas, las bases a sentar durante la edad temprana constituyen un elemento fundamental en el desarrollo gradual de las destrezas para el buen uso y aplicación de las tecnologías durante la experiencia escolar así como fuera del contexto escolar. Un examen del perfil de competencias en el área de las tecnologías identificadas por ISTE que deben poseer los niños al completar el nivel de cuatro a siete años revela como estas se van adquiriendo en forma progresiva a través de los años denotando la importancia de introducir las mismas desde la edad temprana (ISTE, 2007). Por otro lado, la carencia de experiencias y oportunidades para usar las tecnologías desde los primeros años que experimentan los niños con escasos recursos 
económicos se ha encontrado es uno de los elementos que contribuye a la llamada división digital la cual coloca en desventaja a quienes no tienen las mismas experiencias (NAEYC, 2011). Los esfuerzos para eliminar y evitar estas diferencias sirven también para justificar la necesidad para su incorporación en las experiencias de los niños durante los primeros años. Sin duda alguna, las evidencias permiten afirmar que las tecnologías pertenecen y tienen su lugar en el espacio del aula del nivel infantil.

Si bien afirmamos que las tecnologías son parte de las experiencias en la sala de clases del nivel infantil, también es necesario señalar que su uso debe adecuarse a las necesidades y realidades de desarrollo de los niños. Es justamente este aspecto donde es necesario precisar las pautas y parámetros a seguir para su incorporación en el aula infantil. Su uso implica una cuidadosa selección así como planificación por parte del educador que permita su incorporación y aplicación efectiva siendo este punto, en efecto, lo que constituye el mayor reto para su uso en la sala de clases con los niños pequeños. La relevancia de este aspecto es una de las consideraciones señaladas en común a través de los estudios realizados sobre el uso de las tecnologías en el aula infantil (Cause y Chen, 2010; Lee, 2009; Hinchliff, 2008; Gimbert, B. y Cristol, 2004) así como por las organizaciones profesionales con atención a la niñez (NAEYC, 2012; Asociación Americana de Pediatría, 2011). Todos coinciden en destacar la función del educador infantil cuyo criterio profesional debe guiar la selección y forma de uso en el aula. Con el docente como mediador, las tecnologías proporcionan un medio activo para estimular la exploración fomentando el desarrollo cognitivo así como facilitan la interacción social al compartir juegos y materiales en el aula. Su buen uso nos recuerda como mencionáramos antes que en ningún momento las tecnologías substituyen el papel que juega el maestro como facilitador del aprendizaje (Haugland, 2000). El buen juicio del educador se impone como un elemento clave en la planificación y modo de utilización siempre de forma activa y nunca como un medio pasivo. Se impone aquí el rigor del conocimiento del docente sobre las formas de aprender de los niños como manera para diligentemente integrar las tecnologías de manera positiva y apropiada.

\section{Capacitación y formación como clave para el buen uso}

Teniendo en cuenta el rol que tiene el educador infantil en el uso de las tecnologías con los niños, reconocemos que en algunos casos es precisamente este aspecto donde se apoya la contención de algunos sobre su incorporación donde continúa teniéndose como argumento el pobre uso que lamentablemente ha sido observado en algunas aulas que ya sea incluyen algún equipo pero que no es utilizado o que están faltos del conocimiento para su buena integración en el aula (NAEYC, 2011). Sin duda, este hecho revela la necesidad de formación donde se prepare al maestro respecto a su función, rol y forma de empleo como parte de las experiencias de aprendizaje, siendo esta hoy en general una expectativa del perfil del maestro (NAEYC, 2011; UNESCO 2011; 2003). Evidentemente este aspecto subraya la importancia de integrar las tecnologías como parte de las estrategias en la formación profesional del maestro así como tema dentro del plan de estudios, lo cual ya está presente en muchos programas de preparación para el magisterio (ISTE, 2007). Las experiencias durante la formación profesional ofrecen oportunidades para experimentar y conocer las ventajas y posibilidades de las tecnologías, habilidad que sin duda contribuye a su integración en el aula. En entrevis- 
tas realizadas con maestros del nivel infantil que asistían a un programa de capacitación donde se usaba la modalidad combinada de enseñanza presencial y a distancia se encontró que algunos poseían poca o ninguna experiencia en el uso de las computadoras. Superada su aprensión inicial, la práctica y experiencias graduales con las plataformas de enseñanza al igual que con los sistemas digitales y recursos virtuales resultó ser uno de los aspectos que subrayaran como más valiosos en el programa. Algunos señalaron como el interés desarrollado les había motivado a adquirir computadoras personales. Otros señalaron como en sus aulas habían comenzado a planificar de forma consistente experiencias en el rincón de computadoras relacionado con los temas que exploraban los niños (Robles de Meléndez y Valdés, 2011).

\section{Prácticas apropiadas y buen conocimiento, claves para su uso efectivo}

Planteado su lugar en el aula infantil con atención a educar con miras a lo que la sociedad actual y del futuro requiere, es necesario contar con pautas a seguir para el uso de las tecnologías. En el establecimiento de los parámetros a seguir en la práctica pedagógica vemos tres aspectos esenciales a considerar: el conocimiento cabal sobre los niños y sus necesidades de desarrollo, la alineación con expectativas curriculares y el rol que tiene el maestro. Este último como hemos indicado anteriormente, es vital y sirve como eje en la integración de las tecnologías como parte del currículo (NAEYC, 2012; Lee, 2009; Clements y Sarama, 2003).

Como primer aspecto, visto desde el punto de vista de la práctica pedagógica, es necesario conocer ante todo las características de los usuarios, en este caso los niños durante la edad temprana y que aquí vemos como desde el nacimiento hasta los ocho años. Apoyar y contribuir al desarrollo integral con atención a cada uno de los diferentes dimensiones del mismo -cognitivas, sociales, lenguaje y físicas- es el elemento central que sirve de guía y orienta la metas programáticas en el nivel infantil. Es por tanto esencial tener un concepto claro de aquellos elementos que bien describen las capacidades y habilidades en proceso de desarrollo que experimentan los niños en los primeros años. Su conocimiento permite en todo momento adecuar las decisiones educativas, en este caso, el uso cuidadoso e integración de las tecnologías, a la realidad de lo que el niño puede hacer de acuerdo a cada estadio de su desarrollo. Con esta idea como eje, es aquí donde el concepto de prácticas adecuadas según el desarrollo (Bredekamp, y Copple, 2009) proporciona un marco para el uso y determinación de los propósitos a seguir al integrar las tecnologías. Fundamentado en tres elementos claves, el concepto subraya los siguientes como necesarios al considerar la planificación de experiencias en el aula infantil:

- Lo que sabemos sobre las características universales del desarrollo que permiten identificar en forma general lo que pueden hacer los niños de acuerdo a cada periodo del desarrollo durante los primeros ocho años.

- Las características individuales que poseen los niños y que establecen su particularidad

- Las experiencias que poseen los niños moduladas por su entorno cultural y el ámbito de la familia 
En cada uno de estos puntos el niño y su desarrollo general e individual sirven como el elemento clave asegurando así que la integración de tecnologías responda a sus necesidades al igual que a las metas y aspiraciones de la educación temprana. Constatar por tanto como las mismas se acomodan a las características individuales nos lleva en todo momento a preguntarnos (NAEYC, 1996, 2011; Robles de Meléndez y Valdés, 2008):

- ¿En qué forma contribuirán las tecnologías al desarrollo del niño?

- ¿Acaso el uso de las mismas está de acuerdo a los niveles de desarrollo del niño, es decir, a lo que puede hacer?

- ¿Permiten éstas un sentido de éxito y logro en el niño?

- ¿En qué manera se apoya el desarrollo de las destrezas y habilidades del niño?

- ¿Cuáles son las ganancias intelectuales y sociales? ¿Cuáles fomenta el uso de las tecnologías seleccionadas?

- En su uso, ¿se respeta la forma de aprender propia de los niños? Por ejemplo, ¿se facilita su exploración y se incorpora el elemento del juego en su uso?

- Respecto a la selección de recursos y programas, ¿acaso son estos culturalmente apropiados a la realidad de los niños? ¿están estos libres de prejuicios y estereotipos (información, datos o representaciones)?

En función de su uso adecuado tener una clara concepción del propósito y razón de ser del rol de las tecnologías dentro de las experiencias curriculares viene a ser primordial, lo cual apunta al segundo aspecto que debemos considerar. Se acentúa aquí la importancia de conocer cabalmente las expectativas del currículo y como el tipo de interacción con las tecnologías puede contribuir al logro de sus metas. Ello nos lleva a contestar cómo y para que fin se emplean las tecnologías lo cual establece su propósito con fines de aprendizaje específico. Se disipa así toda oportunidad del uso por meramente su uso cuyo fin queda pues en el vacío, algo como mencionaremos antes observado y señalado como argumento en contra por aquellos que dudan de su rol en el aula infantil. En este aspecto vemos a las tecnologías como un recurso que permita enriquecer las experiencias incrementando así las oportunidades para apoyar el aprendizaje de acuerdo a los parámetros de desarrollo general y particular de los niños. Su buen uso comienza teniendo siempre en cuenta que los pequeños requieren de un programa bien balanceado donde se respete la forma en que estos aprenden (Bredekamp y Copple, 2009; NAEYC, 2011). Según NAEYC (2011) tomar decisiones efectivas para su utilización de las tecnologías abarca aspectos sobre su selección, el tipo de uso, su forma y manera de integración así como su evaluación cuidadosa.

El rol del maestro en la selección y determinación de las tecnologías a emplear en el aula es el tercer aspecto a considerar. Como elemento esencial en el uso efectivo se plantea el conocimiento especifico sobre el papel que tienen las tecnologías en el aula así como su justificación dentro del aula infantil. En este aspecto es importante señalar que ya hoy el manejo y conocimiento pedagógico sobre las tecnologías en la educación se incluye en las competencias que definen al educador infantil (NAEYC, 2009). El conocimiento profesional sobre la fundamentación que apoya la integración apropiada 
y presencia de las tecnologías sirve de marco para su selección y aplicación. Dado el creciente número de datos sobre su efectividad en el aula para el docente esto implica además la necesidad de actualizar conceptos de forma constante. Igualmente relevante es la experiencia concreta que tenga el docente en el uso de las diferentes tecnologías. Sin duda, para aquellos que ya son usuarios de las tecnologías su experiencia ofrece ventajas en la selección de cuáles a utilizar. Junto al conocimiento profesional docente la experiencia que se tenga servirá como guía en la determinación de lo que es más adecuado para el nivel y objetivo que se persiga. La oferta de oportunidades para ganar experiencias es clave para llevar a aquellos menos experimentados a ver su valor y uso. Experiencias con grupos de docentes del nivel infantil revelan que en muchos casos cuando se facilita su uso, aun aquellos que expresaron temor a usarlos superaron el mismo al poder experimentar con una selección de tecnologías (Robles de Meléndez y Valdés, 2011). Su práctica sin duda es la mejor forma de conocer sus beneficios y maneras de integración en el aula.

En un mundo donde la presencia y uso de las tecnologías ya viene a ser parte de la rutina del diario vivir no hay duda de su influencia y relevancia. Desde el punto de vista de los educadores su uso responsable continúa siendo una gran preocupación. La misma subraya particularmente la necesidad de ampliar las investigaciones sobre sus efectos y prácticas. Es tal vez en la dimensión empírica donde más necesidad existe sobre todo en términos de estudios investigativos con atención a la población infantil.

\section{Reflexiones finales}

"La tecnología llegó para quedarse", es una frase que por años ha sido repetida por tantos y la cual otros quizás consideraron como algo a ser realidad en el futuro. Sin embargo, el futuro es hoy. La diaria convivencia en la sociedad es donde tal vez mejor entendemos la resonancia e importancia que hoy ya tienen las tecnologías. Basta sólo con salir a la calle y observar el uso de celulares incluyendo aquellos con acceso a Internet así como las tabletas, ipods y juegos digitales como algo ya común. Su presencia podemos afirmar abarca el mundo de todos y se subraya en el ámbito de los niños donde los juguetes electrónicos y digitales han establecido nuevas concepciones sobre el juego. Igualmente notamos como se refleja la presencia de las tecnologías en las frases y vocablos que escuchamos y que tal vez usamos en el diario conversar (por ejemplo, chatear, Tweeter, Wifi, entre tantas otras). Y es que hoy al hablar de aquellos detalles que describen a nuestra sociedad no podemos perder de vista que en éstas se encuentran sin duda la existencia de las tecnologías. Aún más, ya hoy, como expectativa individual su uso apropiado y de forma efectiva se suma a la lista de destrezas y habilidades que la sociedad considera como esenciales para todo individuo. Mucho más, vemos como ya conocer y usar las tecnologías forman parte de lo que algunos consideran uno de los elementos clave en la denominada "ciudadanía digital" (Ribble, 2011). Desde las más simples a las más sofisticadas, las tecnologías son un elemento vital del diario vivir porque sin duda, llegaron y están aquí.

Como bien reflejan las experiencias y estudios la buena utilización de las tecnologías en el nivel infantil indudablemente depende de varios factores. En su buen empleo nos parece importante considerar los siguientes puntos: 
- Conocimiento de los niños y sus características del desarrollo: Saber y tener un conocimiento claro sobre los niños con atención a las características individuales del grupo de niños con las cuales se emplearán; Considerar aquellas que mejor respondan a los niños.

- Experiencia y conocimiento profesional sobre las tecnologías: cuánto conozco sobre las tecnologías, cuál es mi experiencia como usuario; que debo conocer antes de integrarlas.

- Fines para su integración y uso: cómo proponemos emplearlas, cuáles son sus propósitos dentro de las experiencias curriculares, cómo contribuirán al aprendizaje de los niños.

- Expectativas y roles: al emplearlas, qué espero de los niños; cuál será su rol; cómo veo mi rol como maestro.

- Reflexión y valoración: como sabré que las mismas lograron los fines y propósitos igualmente como cuales fueron más efectivas.

Llegaron y permanecen como elemento que forma parte de nuestra identidad social. Así podemos resumir la presencia de las tecnologías en todos los ángulos de nuestra vida. En el aula infantil, donde los niños exploran y se preparan para recorrer el camino del mundo escolar, la integración y buen uso de las tecnologías contribuyen al logro del compromiso educativo que con celo realiza el educador infantil.

\section{Referencias bibliográficas}

AMERICAN ACADEMY OF PEDIATRICS [ASOCIACIÓN AMERICANA DE PEDIATRÍA]. (2011). Policy statement. Media use by children younger than two. Council on Communications and Media. Pediatrics. Obtenido en: http://pediatrics.aappublications.org/content/early/2011/10/12/peds.2011-1753

BREDEKAMP, S. \& COPPLE, C. (2009). Developmentally appropriate practice in early childhood programs. (3ra edición). Washington, DC: National Association for the Education of Young Children.

CAUSE, L. \& CHEN, D. (2010). A tablet computer for young children? Exploring its viability for early childhood education. Journal of Research on Technology in Education, 43 (1), 75-98.

CLEMENTS, D \& SARAMA, J. (2003). Young children and technology: What does the research say? Young Children, 58 (6), 34-40.

ESTHET-ALKALT, \& CHAJUT, E. (2007). Living books: The incidental bonus of playing with multimedia. Journal of Educational Multimedia and Hypermedia 16 (4), 377-388.

FISCH, S. \& TRUGLIO, R. T. (2001). "G" is for growing: Thirty years of research on children and Sesame Street. Mahwah, NJ: Erlbaum.

FISCH, S. (2004). Children's learning from educational television: Sesame Street and beyond. Mahwah, NT: Erlbaum. 
GIMBERT, B. \& CRISTOL, D. (2004). Teaching curriculum with technology: Enhancing children's technological competence during early childhood. Early Childhood Education Journal 31 (3), 207-216.

HAUGLAND, S. (2000). Early childhood classrooms in the 21st century: Using computers to maximize learning. Young Children, 55 (1), 12-18.

HAUGLAND, S. (1999). What role should technology play in young children's leaning? Part I. Young Children 54 (6), 26-31.

HINCHLIFF, G. (2008). Toddling toward technology: Computer use by very young children. Children and Libraries 6 (3), 47-49.

INTERNATIONAL SOCIETY FOR TECHNOLOGY IN EDUCATION [ISTE]. (2007). Profile for technology literate students (PreK-2). Obtenido en http://www.iste.org/Libraries/PDFs/NETS_for_Student_2007_EN.sflb.ashx

JOHNSON, D. (2011). Wired. AAP News, Vol. 32, Num. 4, p.1.

JUDGE, S. (2005). The impact of computer technology on academic achievement of young African-American children. Journal of Research in Childhood Education 20 (2), 91-101.

KAISER FAMILY FOUNDATION Y THE CENTER ON MEDIA AND CHILD HEALTH DEL CHILDREN'S HOSPITAL BOSTON. (Enero 2005). The effects of electronic media on children zero to six: A history of research. Issue Brief \# 7239. Washington, DC: The Henry Kaiser Family Foundation.

LAU, C., HIGGINS, K., HONG, E. \& MILLER, S. (2005). The effects of teacher facilitation on the social interactions of young children during computer activities. Topics in early Childhood Special Education 25 (4), pp. 208-217.

LISENBEE, P. (2009). Whiteboards and web sites: Digital tools for the early childhood curriculum. Young Children 64 (5), 92-95.

NATIONAL ASSOCIATION FOR THE EDUCATION OF YOUNG CHILDREN. (2011). Technology in early childhood programs serving children from birth through age 8. (Borrador). Washington, DC: Autor.

NATIONAL ASSOCIATION FOR THE EDUCATION OF YOUNG CHILDREN. (2012 ). Technology and interactive media in early childhood programs serving children from birth through age 8. A joint statement of the National Association for the Education of Young Children and the Fred Rogers Center for Early Learning and Children's Media at Saint Vincent College. Washington, DC: Autor

NATIONAL ASSOCIATION FOR THE EDUCATION OF YOUNG CHILDREN. (2009). Standards for early childhood teacher preparation: a position statement of the National Association for the Education of Young Children. Obtenido en: http://www,naeyc.org/files/naeyc/file/positions/ProfPrepStandards09.pdf

NATIONAL ASSOCIATION FOR THE EDUCATION OF YOUNG CHILDREN. (1996). Position Statement. Technology in early childhood programs serving children from birth through age 8. Washington, DC: Autor. 
O'KEEFE, G \& CLARK-PEARSON, K. (2011). The impact of social media on children, adolescents and families. Clinical report. Pediatrics, Vol. 127, Num. 4, pp. 800-804.

RIBBLE, M. (2011). Digital citizenship in schools. (2 ${ }^{\text {nd }}$. ed.). Washington, DC: International Society for Technology in Education [ISTE].

Rideout, V, Vandewater, E. \& Wartella, E. (2003). Zero to six. Electronic media in the lives of infants, toddlers and preschoolers. A Kaiser Family Foundation report. Washington, DC: The Henry Kaiser Family Foundation.

ROBLES DE MELÉNDEZ, W. \& VALDÉS, M. (2008). El juego y el uso de las tecnologías. Fort Lauderdale, FL: Taller de trabajo en el Congreso de Nova Educa.

ROBLES DE MELÉNDEZ, W. \& VALDÉS, M. (2011). Crecer para hacer crecer. Un modelo bilingüe para apoyar el desarrollo de los docentes del nivel infantil. Orlando, FL: Presentación en la Conferencia anual de la National Association for the Education of Young Children.

STRASSBURGER, V. (2010). Children, adolescents, and the media: Seven key issues. Pediatric Annals, 39 (9), 556-564.

STRICKLAND, D., \& RATH, L. K. (2000). "Between the lions": Public television promotes early literacy. Newark, DE: International Reading Association. (ED 444 118).

THAKKAR, GARRISON \& CHRISTAKIS, (2006). A Systematic Review for the Effects of Television Viewing by Infants and Preschoolers. Pediatrics, 118 (5), 2025-2031.

TURJA, L., ENDEPOHLS-ULPE, M \& CHATONEY, M. (2009). International Journal of Technology and Design Education 19, 353-365.

UNESCO. (2003). Information and communication technologies in teacher education. A planning guide. Obtenido de: http://unesdoc.unesco.org/images/0012/001295/129533e.pdf

UNESCO. (2011). ICT in education strategy. Obtenido de: http://www.unesco.org/new/en/unesco/themes/icts/strategy/

ZEHR, M. (2009). Pre-K Lessons Linked To TV Produce Gains In Literacy, Study Says. Education Week, 29 (8), 9.

\section{Correspondencia con la autora}

Wilma Robles-Meléndez

Abraham S. Fischler School of Education

Nova Southeastern University

1750 NE 167th Street

North Miami Beach, FL 33162 USA

E-mail: martrobw@fse.nova.edu 